\title{
Equações volumétricas para um clone jovem de eucalipto no Sul do Tocantins
}

\author{
Valdir Carlos Lima de ANDRADE ${ }^{1 *}$, Murilo Azevedo GLÓRIA JUNIOR ${ }^{1}$, \\ David Lucas Camargo Vieira TERRA ${ }^{1}$
}

\author{
'Programa de Pós-Graduação em Ciências Florestais e Ambientais, Universidade Federal do Tocantins, Gurupi, TO, Brasil. \\ *E-mail: vclandrade@uft.edu.br
}

Recebido em janeiro/2018; Aceito em outubro/2018

\begin{abstract}
RESUMO: Este trabalho teve como objetivo avaliar um total de 26 modelos volumétricos para um clone do híbrido entre Eucalyptus urophylla e Eucalyptus grandis. Foram utilizados dados da cubagem rigorosa de 25 árvores, distribuídas em cinco classes diamétricas com amplitude de $2 \mathrm{~cm}$ cada. Para a avaliação dos modelos volumétricos, adotou-se os critérios: coeficiente de determinação ajustado, erro padrão residual, análise da distribuição de resíduos, soma dos quadrados dos resíduos relativos, erro médio e raiz do quadrado médio. $\mathrm{O}$ modelo logarítmo: $\operatorname{Ln}(\mathrm{v})=\beta_{0}+\beta_{1} \operatorname{Ln}(\mathrm{d})+\beta_{2} \operatorname{Ln}(\mathrm{h})+\operatorname{Ln}(\varepsilon)$, um dos mais utilizados no Brasil, teve o oitavo lugar como a melhor colocação dentre os 26 modelos volumétricos avaliados. $\mathrm{O}$ modelo que mais se destacou foi: $\operatorname{Ln}(\mathrm{v})=\beta_{0}+\beta_{1} \operatorname{Ln}(\mathrm{d}+1)+\beta_{2} \operatorname{Ln}(\mathrm{h})+\operatorname{Ln}(\varepsilon)$ que, ajustado com a inclusão da variável binária $\mathrm{Tx}$, permitiu o ajuste de uma única equação para quantificar, tanto o volume com casca como sem casca, para o clone estudado. A equação obtida foi: $\hat{\mathrm{v}}=\mathrm{e}^{[1,286 \operatorname{Ln}(\mathrm{d}+1)+2,105 \operatorname{Ln}(\mathrm{h})-11,700-0,167(\mathrm{Tx})]}$.
\end{abstract}

Palavras-chave: inventário florestal, modelos volumétricos, eucalipto.

\section{Volumetric equations for a young eucalyptus clone in the south of Tocantins}

\begin{abstract}
This work aimed to evaluate a total of 26 volumetric models for clone of the hybrid between Eucalyptus urophylla and Eucalyptus grandis. We used data from the rigorous cubing of 25 trees distributed in five diameter classes with amplitude of $2 \mathrm{~cm}$ each. For the evaluation of the volumetric models, the following criteria were adopted: adjusted coefficient of determination, residual standard error, residue distribution analysis, sum of squares of relative residues, mean error and root mean square. The logarithm model: $\operatorname{Ln}(v)=\beta_{0}+\beta_{1} \operatorname{Ln}(\mathrm{d})+\beta_{2} \operatorname{Ln}(\mathrm{h})+\operatorname{Ln}(\varepsilon)$, one of the most used in Brazil, ranked eighth as the best placement among the 26 volumetric models evaluated. The best that most stood out was: $\operatorname{Ln}(\mathrm{v})=\beta_{0}+$ $\beta_{1} \operatorname{Ln}(\mathrm{d}+1)+\beta_{2} \operatorname{Ln}(\mathrm{h})+\operatorname{Ln}(\varepsilon)$ which, adjusted with the inclusion of the binary variable Tx, allowed the adjustment of a single equation to quantify both the shell and shelled volume for the studied clone. The equation obtained was: $\hat{\mathrm{v}}=\mathrm{e}^{[1,286 \operatorname{Ln}(\mathrm{d}+1)+2,105 \operatorname{Ln}(\mathrm{h})-11,700-0,167(\mathrm{Tx})]}$.
\end{abstract}

Keywords: forest inventory, volumetric models, eucalyptus.

\section{INTRODUÇÃO}

Segundo a Indústria Brasileira de Árvores (IBÁ, 2017), a área de plantios florestais brasileiros totalizou 7,84 milhões de hectares (ha) e corresponde a mais de $90 \%$ do uso madeireiro no país. Desses 7,84 milhões de ha, cerca de 5,7 milhões correspondem aos plantios com espécies do gênero Eucalyptus, onde o estado do Tocantins concentra cerca de 120 mil ha (IBÁ, 2017).

Apesar da pouca área plantada no Tocantins, em relação ao total no Brasil, a avaliação quantitativa em estoque se faz necessária para balizar a tomada de decisões e garantir melhor administração de sua produção madeireira. Para isso, é de grande importância realizar estudos sobre volumetria nos plantios florestais do Tocantins onde ainda são muito escassos.

Estudos sobre volumetria envolvem a predição presente e futura feita a partir de dados advindos de um inventário florestal. Na predição futura, onde se faz a prognose do crescimento e produção florestal, é necessário ter algumas predições iniciais de parcelas permanentes medidas anualmente na área de plantio inventariada. Isso exige obter o volume de madeira com as árvores em pé desde a idade mais jovem, geralmente, desde a idade de 2 a 3 anos para eucalipto. Diante deste cenário, caracteriza-se uma situação que exige o emprego de equações volumétricas geradas a partir de modelos estatísticos que relacionam o volume real com variáveis independentes de fácil medição, tais como: altura e, ou, diâmetro das árvores. Para isso, são empregados dados da cubagem de algumas árvores, geralmente, abatidas em locais diferentes das parcelas permanentes (Andrade et al., 2006).

Muitos modelos estatísticos, denominados de modelos volumétricos, já foram testados no Brasil para o ajuste de equações de volume, conforme pode ser notado nos trabalhos de Couto; Vettorazzo (1999), Veiga et al. (2000), Machado et al. (2002), Rezende et al. (2006), Imaña-Encinas et al. (2009), Rocha et al. (2010), Pelissari et al. (2011), Miguel; Leal (2012), dentre outros. Nesses estudos, também, se observam que é mais adequado o uso de modelos volumétricos regionais, que relacionam o volume com o diâmetro e a altura das árvores, do que o uso de modelos volumétricos locais, que relacionam o volume somente com o diâmetro das árvores.

Outra situação é a que se refere ao ajuste de equações volumétricas diferentes para volume com casca e sem casca, gerando-se então duas equações volumétricas, sendo uma 
para estimar o volume com casca e outra para volume da madeira sem a casca. Como resultado, dado à variação de diferentes situações que comprometem a aplicabilidade das equações volumétricas, quais sejam: idade, espaçamento, local, solo, espécie, material genético, dentre outros, gera-se uma grande quantidade de equações volumétricas para serem utilizadas no processamento de um determinado inventário florestal.

Do problema em referência, observa-se que uma possível solução seria a de estimar equações volumétricas para volume com e sem casca a partir do mesmo ajuste, porque vai reduzir em $50 \%$ a quantidade de equações volumétricas necessárias à adequação das diferentes situações já citadas anteriormente. Assim, observa-se ser oportuno testar o emprego da variável binária $\mathrm{Tx}$, a qual é implementada no mesmo modelo volumétrico assumindo: valor 0 para o volume com casca e valor 1 para o volume sem casca. Com isso, pode-se gerar uma única equação volumétrica para quantificar tanto o volume com casca como sem casca por árvore individual (LEITE et al., 1995; CAMPOS; LEITE, 2013).

Diante destas considerações, foram enunciadas para este estudo, as seguintes hipóteses teóricas:

Hipótese 1:

$\mathrm{Ho}_{1}$ : para um clone jovem de eucalipto no sul do Tocantins, modelos volumétricos regionais geram equações com melhor qualidade de ajuste e nível de precisão do que modelos volumétricos locais.

$\mathrm{Ha}_{1}$ : não $\mathrm{Ho}_{1}$.

Hipótese 2:

$\mathrm{Ho}_{2}$ : para um clone jovem de eucalipto no sul do Tocantins, pode-se utilizar uma única equação para quantificar o volume de madeira com e sem casca aplicando a variável binária $\mathrm{Tx}$, ao invés de se utilizar duas equações volumétricas como se faz usualmente, separadamente, uma para volume de madeira com casca e outra para madeira sem casca.

$\mathrm{Ha}_{2}$ : não $\mathrm{Ho}_{2}$.

Portanto, justifica-se que seja conduzido este estudo sobre volumetria de clones jovens de eucalipto no Tocantins, com o objetivo de avaliar as hipóteses teóricas 1 e 2.

\section{MATERIAL E MÉTODOS}

2.1. Localização da área, coleta de dados e modelos volumétricos avaliados

Como estudo de caso, os dados utilizados neste trabalho foram obtidos em um plantio jovem do clone GG100 (híbrido entre Eucalyptus urophylla X Eucalyptus grandis) localizado na região Sul do estado do Tocantins, próximo ao município de Gurupi. Esta região, segundo SEPLAN (2012), além de uma precipitação média de $1.500 \mathrm{~mm} / \mathrm{ano}$, tem clima predominante do tipo $\mathrm{C}_{2} \mathrm{w} 2 \mathrm{~A}^{\prime} \mathrm{a}^{\prime}$ da classificação de Thornthwaite-Mather, com duas estações bem definidas: seca no inverno (entre maio a outubro) e úmida no verão (entre outubro e maio).

No plantio de GG100, formado no espaçamento inicial de $6,0 \times 1,5 \mathrm{~m}$ e com cerca de 3 anos de idade, foi implementado um inventário florestal contínuo para acompanhar o seu desenvolvimento em volume de madeira na região sul do Tocantins. Neste caso, para quantificar o volume das árvores em pé, foi necessário empregar equações volumétricas geradas com dados advindos de uma cubagem rigorosa (ANDRADE et al., 2006; MACHADO; FIGUEIREDOFILHO, 2006).
$\mathrm{Na}$ cubagem rigorosa realizada, por se tratar de um clone jovem (3 anos) e de pouca variação diamétrica, foram abatidas 25 árvores distribuídas em classes de diâmetro com amplitude de $2 \mathrm{~cm}$ e tendo cinco árvores cada (Tabela 1), conforme número recomendado para eucalipto, entre 5 a 7 indivíduos por classe diamétrica (ANDRADE; LEITE, 2001). Em seguida, após o abate de cada árvore, foram feitas medições do diâmetro do tronco com e sem casca nas posições: $0,1 \mathrm{~m}, 0,20 \mathrm{~m}, 0,40 \mathrm{~m}, 0,70 \mathrm{~m}, 1,30 \mathrm{~m}, 2,70 \mathrm{~m}$ e, sucessivamente, de $2 \mathrm{~m}$ em $2 \mathrm{~m}$ até um diâmetro de $3 \mathrm{~cm}$ com casca (MACHADO; FIGUEIREDO-FILHO, 2006; CAMPOS; LEITE, 2013).

Tabela 1. Distribuição de frequência de 25 árvores cubadas do clone GG100 no sul do Tocantins, Brasil.

Table 1. Frequency distribution of 25 cubed trees of clone GG100 in southern Tocantins, Brazil.

\begin{tabular}{cccccccc}
\hline Classe & \multicolumn{7}{c}{ Classe de altura $(\mathrm{m})$} \\
\cline { 2 - 7 } $\mathrm{DAP}(\mathrm{cm})$ & 5 & 7 & 9 & 11 & 15 & 17 & Total \\
\hline 4 & 1 & 4 & & & & & 5 \\
6 & & & 2 & 3 & & & 5 \\
8 & & & & 2 & 2 & 1 & 5 \\
10 & & & & & & 5 & 5 \\
12 & & & & & & 5 & 5 \\
\hline Total & 1 & 4 & 2 & 5 & 2 & 11 & 25 \\
\hline
\end{tabular}

Os dados de cubagem foram obtidos de forma à facilitar a aplicação da fórmula de Huber para obter o volume do fuste que, acrescido dos volumes da ponta (cone) e da cepa $(0,10)$, resultou no volume total com e sem casca de cada árvore (MACHADO; FIGUEIREDO-FILHO, 2006; CAMPOS; LEITE, 2013). O valor obtido foi utilizado no ajuste dos seguintes modelos volumétricos, conforme já testados no Brasil por Campos et al. (1985), Couto; Vettorazzo (1999), Veiga et al. (2000), Machado et al. (2002), Machado et al. (2008), Imaña-Encinas et al. (2009), Rocha et al. (2010), Pelissari et al., (2011), Andrade; Schmitt (2016) e Andrade (2017):

$$
\begin{aligned}
& \mathrm{v}=\beta_{0}+\beta_{1} d+\beta_{2} d^{2}+\beta_{3} d h+\beta_{4} d^{2} h+\varepsilon(1) \\
& \operatorname{Ln}(v)=\beta_{0}+\beta_{1} \operatorname{Ln}(d)+\beta_{2} \operatorname{Ln}(h)+\operatorname{Ln}(\varepsilon)(2) \\
& v=\frac{d^{2} h}{\beta_{0}+\beta_{1} d}+\varepsilon(3) \\
& v=\beta_{0}+\beta_{1} d^{2}+\beta_{2} \operatorname{Ln}\left(d^{2} h\right)+\beta_{3}\left(d^{2}\right)+\beta_{4} h^{2}+\varepsilon(4) \\
& v=\beta_{0}+\beta_{1}\left(d^{2} h\right)+\varepsilon(5) \\
& \operatorname{Ln}(v)=\beta_{0}+\beta_{1} \operatorname{Ln}\left(d^{2} h\right)+\operatorname{Ln}(\varepsilon)(6) \\
& v=\beta_{0}+\beta_{1} d+\beta_{2} d h+\beta_{3} d^{2}+\beta_{4} h \\
& \quad+\beta_{5}\left(d^{2} h\right)+\varepsilon(7) \\
& \operatorname{Ln}(v)=\beta_{0}+\beta_{1} \operatorname{Ln}\left(d h^{2}\right)+\operatorname{Ln}(\varepsilon) \\
& v=\frac{d^{2}}{\beta_{0}+\beta_{1}\left(\frac{1}{h}\right)}+\varepsilon(9) \\
& v=d^{2}\left(\beta_{0}+\beta_{1} h\right)+\varepsilon(10) \\
& \operatorname{Ln}(v)=\beta_{0}+\beta_{1} \operatorname{Ln}(d)+\left(3-\beta_{1}\right) \operatorname{Ln}(h) \\
& \quad+\operatorname{Ln}^{2}(\varepsilon)(11) \\
& \operatorname{Ln}(v)=\beta_{0}+\beta_{1} \operatorname{Ln}(d+1)+\beta_{2} \operatorname{Ln}(h)+\operatorname{Ln}(\varepsilon)(12) \\
& \operatorname{Ln}(v)=\beta_{0}+\beta_{1} \operatorname{Ln}\left(\frac{h}{d}\right)+\operatorname{Ln}\left(d^{2} h\right)+\operatorname{Ln}(\varepsilon)(13) \\
& \operatorname{Ln}(v)=\beta_{0}+\beta_{1} \operatorname{Ln}(d)+\beta_{2}(\operatorname{Lnd})^{2}+\beta_{3} \operatorname{Ln}(h)+ \\
& \quad \quad \beta_{4}(\operatorname{Lnh})^{2}+\operatorname{Ln}(\varepsilon)(14) \\
& v=\beta_{0}+\beta_{1} d^{2}+\beta_{2}\left(d^{2} h\right)+\beta_{3} h+\varepsilon(15) \\
& v=\beta_{1} d^{\beta_{2}} h^{\beta_{3}}+\varepsilon(16) \\
& v=\beta_{1}\left(d^{2} h\right)^{\beta_{2}}+\varepsilon(17) \\
& v=\beta_{1}+\beta_{2}\left(d^{2} h\right)+\beta_{3}\left(d h^{2}\right)+\varepsilon(18) \\
& \operatorname{Ln}(v)=\beta_{0}+\beta_{1} \operatorname{Ln}(d)+\operatorname{Ln}(\varepsilon)(19)
\end{aligned}
$$


$\operatorname{Ln}(v)=\beta_{0}+\beta_{1} \operatorname{Ln}(d)+\beta_{2}\left(\frac{1}{d}\right)+\operatorname{Ln}(\varepsilon)(20)$

$\operatorname{Ln}(\mathrm{v})=\beta_{0}+\beta_{1} \operatorname{Ln}(\mathrm{d})+\beta_{2}(\operatorname{Ln} \mathrm{d})^{2}+\operatorname{Ln}(\varepsilon)(21)$

$\mathrm{v}=\beta_{0}+\beta_{1}\left(\frac{1}{\mathrm{~d}}\right)+\beta_{2}\left(\frac{1}{\mathrm{~d}}\right)^{2}+\varepsilon(22)$

$\mathrm{v}=\beta_{0}+\beta_{1} \mathrm{~d}^{2}+\varepsilon(23)$

$\mathrm{v}=\beta_{1} \mathrm{~d}+\beta_{2} \mathrm{~d}^{2}+\varepsilon(24)$

$\mathrm{v}=\beta_{0}+\beta_{1} \mathrm{~d}+\beta_{2} \mathrm{~d}^{2}+\varepsilon(25)$

$v=\beta_{0} d^{\beta_{1}}+\varepsilon(26)$

em que: $\mathrm{d}=\mathrm{DAP}(\mathrm{cm}), \mathrm{h}=$ altura $(\mathrm{m}), \beta_{0}, \beta_{1}, \beta_{2}, \beta_{3}, \beta_{4}, \beta_{5}=$ Coeficientes a serem estimados, $\mathcal{E}=$ Erro do modelo, Ln=Logarítmo neperiano e $\mathrm{v}=\operatorname{volume}$ total $\left(\mathrm{m}^{3}\right)$.

\subsection{Critérios estatísticos de análise}

Utilizando-se o Software R For Windows 3.15.2 (R CORE TEAM, 2015), procedeu-se ao ajuste e avaliação dos 26 modelos volumétricos por meio dos critérios estatísticos: coeficiente de determinação ajustado ( $\left.R^{2} a j\right)$, erro padrão residual (syx), média das diferenças absolutas (MDA), desvio padrão das diferenças (DPD) e soma dos quadrados dos resíduos relativos (SQRR), além da análise da distribuição de resíduos. Neste caso, os procedimentos de análises com estes critérios estatísticos podem ser vistos em: Campos et al. (1985), Couto; Vettorazzo (1999), Machado et al. (2002), Rezende et al. (2006), Rocha et al. (2010), Miguel; Leal (2012), Pelissari et al. (2011) e Andrade; Schmitt (2016).

Inicialmente, a partir de uma análise preliminar com o $\mathrm{R}^{2} \mathrm{aj}$, syx e análise da distribuição de resíduos, selecionou-se os modelos ajustados com a melhor qualidade e nível de precisão. Em seguida, dentre estes melhores modelos, procedeu-se à uma análise detalhada por classe de diâmetro considerando-se as demais estatísticas. Estes critérios, obtidos por classe de diâmetro, conforme Andrade; Schmitt (2016) e Andrade (2017), foram classificados por meio da atribuição de notas em ordem crescente do melhor ao pior modelo. Após isso, as notas obtidas por classe de diâmetro, foram englobadas em um único valor pela soma geral. Tal resultado geral, através do menor valor e aliado à análise da distribuição de resíduos, indicou os melhores modelos volumétricos para o clone GG100 no Sul do Tocantins.

Identificados os modelos de maior destaque, verificou-se as pressuposições de regressão quanto à normalidade, heterocedasticidade e indeendência de resíduos (REGAZZI; SILVA, 2004, SCHENEIDER et al., 2009). Neste caso, considerou-se o teste de Kolmogorov-Smirnov para verificar a distribuição de normalidade, o teste de Bartlett para verificar a ausência de heterocedasticidade e o teste de Durbin-Watson para verificar se os resíduos não estão correlacionados, ou, se são independentes.

O melhor modelo volumétrico foi ajustado para volume sem casca e depois para volume com e sem casca utilizandose a variável binária $T x=0$ para o volume com casca e $T x=1$ para o volume sem casca. Como resultado, obteve-se uma única equação volumétrica para quantificar os volumes com e sem casca por árvore individual do clone GG100. Nesta etapa, as equações para volume com e sem casca do modelo selecionado foram comparadas com a equação gerada empregando-se a variável Tx.

\section{RESULTADOS}

$\mathrm{Na}$ Tabela 2 são apresentados os resultados obtidos pelo ajuste de 26 modelos volumétricos. Nota-se que, enquanto os modelos volumétricos regionais apresentaram $\mathrm{R}^{2}$ aj maior que 0,959 e syx entre $8,6 \%$ a $16,7 \%$, os modelos volumétricos locais apresentaram uma expressiva piora nestes resultados, sendo $\mathrm{R}^{2}$ aj entre 0,924 a 0,942 e syx entre $20,1 \%$ a $23,4 \%$.

Pelos resultados obtidos, houve a seleção dos modelos regionais $1,2,4,7,8,12,13,15,16$ e 18 , sendo o modelo 4 o que apresentou os melhores $\mathrm{R}^{2}$ aj e syx com valores de 0,991 e $8,62 \%$, respectivamente. Esses dez modelos volumétricos, previamente selecionados com base no $R^{2}$ aj e syx, foram submetidos a análise das estatísticas MDA, DPD e SQRR (Tabela 3).

$\mathrm{Na}$ Tabela 3 observa-se não haver um único modelo que seja o melhor para todas as classes diamétricas. Nesse caso, a escolha do melhor modelo se deu a partir do somatório das notas em todas as classes diamétricas, sendo o modelo $12 \mathrm{o}$ de menor nota $(\mathrm{N}=61)$, seguido do modelo 1 com $\mathrm{N}=62$. Já, o modelo de Shumacher e Hall (modelos 2 e 16), um dos mais utilizados no Brasil, se classificou em $8^{\circ}$ e $4^{\circ}$ lugares com 80 e 65 pontos, respectivamente. Portanto, tendo como base os resultados das Tabelas 2 e 3 , os modelos 1 e 12 se sobressaíram no ajuste aos dados do clone GG100.

Ao contrastar as Figuras 1A e 1D, nota-se que o modelo 1 apresentou tendência de subestimativa do volume entre 0,04 $\mathrm{m}^{3}$ e $0,08 \mathrm{~m}^{3}$, o que não se observa ocorrer com o modelo 12 que apresentou a melhor distribuição dos resíduos com $72 \%$ $(18 / 25)$ em $\pm 0,003 \mathrm{~m}^{3}$ contra $64 \%(16 / 25)$ do modelo 1. Já, pelas Figuras 1B e 1E, se observa uma distribuição tendendo à normalidade para ambos os modelos 1 e 12, com melhor distribuição para o modelo 12 com $60 \%$ (15/25) dos resíduos oscilando em torno de $\pm 0,002 \mathrm{~m}^{3}$ contra $48 \%(12 / 25)$ do modelo 1, respectivamente (Figura $1 \mathrm{~F}$ versus Figura 1C).

Embora a análise Figura 1 seja suficiente para validar a pressuposição de normalidade dos resíduos, se observa uma quase imperceptível ocorrência de heterogeneidade de variância, sendo mais pronunciada para o modelo 1 (Figura $1 \mathrm{~A}$ versus Figura 1D). Nesse caso, aplicando-se o teste de Bartlett ao nível de 0,05 de significância, obteve-se valores de 14,65 e 8,10 para os modelos 1 e 12, respectivamente. Tais resultados, comparados ao valor tabelar de 9,49, indicou rejeição da hipótese sobre homogeneidade de variância para 0 modelo 1 e aceitação desta hipótese para o modelo 12, condição desejável para uso da regressão linear (SCHENEIDER et al., 2009).

Por outro lado, aplicando-se o teste de KolmorovSmirnov para os modelos 1 e 12, obteve-se valores de 0,11 e 0,15 . Tais resultados, comparados ao valor tabelado de 0,27 , levou à aceitação da hipótese sobre haver normalidade dos resíduos para ambos os modelos 1 e 12 (SCHENEIDER et al., 2009).

Quanto à independência de resíduos, verificada pelo teste de Durbin-Watson, obteve-se para os modelos 1 e 12 os valores de 2,32 e 1,84, respectivamente. Nesse caso, rejeitouse a hipótese sobre não existir correlação em série nos resíduos para o modelo 1 e aceitou-se esta hipótese para o modelo 12 (SCHENEIDER et al., 2009). Isso indica que os resíduos são dependentes e apresentam uma correlação em série para o modelo 1 e, para o modelo 12 , os resíduos são independentes, condição desejável para o uso da regressão linear. Este resultado, aliado ao que foi obtido pela análise de homocedasticidade, indicou superioridade estatística do modelo 12 frente ao 1 .

A análise feita anteriormente, é complementada pela Figura 2 onde se observam melhores resultados para o 


\section{Equações volumétricas para um clone jovem de eucalipto no Sul do Tocantins}

modelo 12 com menor variação nos resíduos, tanto na amplitude total como no intervalo interquartil, além de uma mediana mais próxima de $0,000 \mathrm{~m}^{3}$. Portanto, como o modelo 12 apresentou os melhores resultados em todas as análises feitas anteriormente frente ao modelo 1, o qual foi superior de forma pouco expressiva apenas quanto ao $R^{2}$ aj e syx(Tabela 2), escolheu-se este modelo para utilizar na área a fim de obter os volumes com e sem casca do clone GG100.
Diante disso, utilizando-se as estimativas da Tabela 2 e as que foram obtidas pelo ajuste com volume sem casca, gerouse as seguintes equações volumétricas regionais:

Volume com casca $\left(v_{c c}\right)$

$\widehat{\mathrm{v}_{\mathrm{cC}}}=\mathrm{e}^{[1,276 \mathrm{Ln}(\mathrm{d}+1)+2,092 \mathrm{Ln}(\mathrm{h})-11,644]} ;$ com $\mathrm{R}^{2} \mathrm{aj}=97,77 \%$ e syx $=$ $12,18 \%$

Volume sem casca $\left(v_{s c}\right)$

(Equação 1)

$\widehat{\mathrm{V}_{\mathrm{SC}}}=\mathrm{e}^{[1,297 \operatorname{Ln}(\mathrm{d}+1)+2,118 \mathrm{Ln}(\mathrm{h})-11,922]} ; \operatorname{comR}^{2} \mathrm{aj}=97,78 \%$ e syx $=$ $12,27 \%$

(Equação 2)

Tabela 2. Resultados obtidos para 26 modelos volumétricos ajustados para o clone GG100 na região Sul do Tocantins.

Table. Results obtained for 26 volumetric models adjusted for clone GG100 in the southern region of Tocantins.

\begin{tabular}{|c|c|c|c|c|c|c|c|c|c|c|}
\hline & $\hat{\beta}_{0}$ & $\hat{\beta}_{1}$ & $\hat{\beta}_{2}$ & $\hat{\beta}_{3}$ & $\hat{\beta}_{4}$ & $\hat{\beta}_{5}$ & $R_{a j}^{2} \%$ & $\operatorname{Syx}\left(\mathrm{m}^{3}\right)$ & Syx \% & $\mathrm{N}$ \\
\hline \multicolumn{11}{|c|}{ MODELOS REGIONAIS } \\
\hline 1 & 0,04742 & $-0,02458$ & $1,51 \mathrm{E}-03$ & $1,32 \mathrm{E}-03$ & $-6,29 \mathrm{E}-05$ & & $98,35^{4}$ & 0,00442 & $11,48^{5}$ & 9 \\
\hline 2 & $-11,128$ & 1,093 & 2,101 & & & & $97,60^{10}$ & 0,00487 & $12,64^{10}$ & 20 \\
\hline 3 & 19913,3 & 733,8 & & & & & $96,52^{13}$ & 0,00598 & $15,54^{13}$ & 26 \\
\hline 4 & 0,1217 & $1,73 \mathrm{E}-03$ & $-0,03844$ & $-8,14 \mathrm{E}-05$ & $1,01 \mathrm{E}-03$ & & $99,07^{1}$ & 0,00332 & $8,62^{1}$ & 2 \\
\hline 5 & $1,14 \mathrm{E}-03$ & $3,49 \mathrm{E}-05$ & & & & & $96,16^{15}$ & 0,00628 & $16,31^{15}$ & 30 \\
\hline 6 & $-10,1519$ & 0,9869 & & & & & $96,00^{17}$ & 0,00628 & $16,31^{15}$ & 32 \\
\hline 7 & 0,02564 & $-0,0318$ & $3,20 \mathrm{E}-04$ & $2,62 \mathrm{E}-03$ & $8,57 \mathrm{E}-03$ & $-6,06 \mathrm{E}-05$ & $98,77^{2}$ & 0,00391 & $10,16^{2}$ & 4 \\
\hline 8 & $-11,155$ & 1,067 & & & & & $97,74^{8}$ & 0,00471 & $12,24^{8}$ & 16 \\
\hline 9 & $-126,5$ & 30141,6 & & & & & $96,12^{16}$ & 0,00632 & $16,41^{16}$ & 32 \\
\hline 10 & 4307,2 & $-158,8$ & & & & & $95,97^{18}$ & 0,00644 & $16,71^{17}$ & 35 \\
\hline 11 & $-10,5$ & 1,42 & & & & & $96,65^{12}$ & 0,00574 & $14,91^{12}$ & 24 \\
\hline 12 & $-11,644$ & 1,276 & 2,092 & & & & $97,77^{7}$ & 0,00469 & $12,18^{7}$ & 14 \\
\hline 13 & $-11,128$ & 1,037 & 1,065 & & & & $97,64^{9}$ & 0,00482 & $12,52^{9}$ & 18 \\
\hline 14 & $-4,344$ & 3,248 & $-0,5423$ & $-5,4473$ & 1,58 & & $97,54^{11}$ & 0,00493 & $12,79^{11}$ & 22 \\
\hline 15 & $-0,01109$ & $-7,80 \mathrm{E}-04$ & $6,76 \mathrm{E}-05$ & $2,80 \mathrm{E}-03$ & & & $98,38^{3}$ & 0,00427 & $11,09^{3}$ & 6 \\
\hline 16 & & $5,71 \mathrm{E}-06$ & 0,8885 & 2,634 & & & $98,25^{5}$ & 0,00433 & $11,25^{4}$ & 9 \\
\hline 17 & & $6,19 \mathrm{E}-05$ & 0,9268 & & & & $96,32^{14}$ & 0,00615 & $15,97^{14}$ & 28 \\
\hline 18 & & $-2,59 \mathrm{E}-03$ & $2,14 \mathrm{E}-07$ & $2,59 \mathrm{E}-05$ & & & $98,01^{6}$ & 0,00462 & $12,01^{6}$ & 12 \\
\hline \multicolumn{11}{|c|}{ MODELOS LOCAIS } \\
\hline 19 & $-9,237$ & 2,729 & & & & & $92,80^{5}$ & 0,00842 & $21,88^{5}$ & 10 \\
\hline 20 & $-9,24123$ & 2,73036 & $9,15 \mathrm{E}-03$ & & & & $92,47^{7}$ & 0,00861 & $22,37^{7}$ & 14 \\
\hline 21 & $-9,37592$ & 2,88371 & $-4,08 \mathrm{E}-02$ & & & & $92,67^{6}$ & 0,00850 & $22,07^{6}$ & 12 \\
\hline 22 & 0,1989 & $-1,7727$ & 3,8508 & & & & $92,42^{8}$ & 0,00902 & $23,44^{8}$ & 16 \\
\hline 23 & $-6,68 \mathrm{E}-03$ & $6,10 \mathrm{E}-04$ & & & & & $94,18^{3}$ & 0,00774 & $20,09^{2}$ & 5 \\
\hline 24 & & $-1,84 \mathrm{E}-03$ & $7,20 \mathrm{E}-04$ & & & & $94,19^{2}$ & 0,00773 & $20,08^{1}$ & 3 \\
\hline 25 & $-3,17 \mathrm{E}-03$ & $-9,90 \mathrm{E}-04$ & $6,70 \mathrm{E}-04$ & & & & $94,20^{1}$ & 0,00789 & $20,50^{4}$ & 5 \\
\hline 26 & $2,20 \mathrm{E}-04$ & 2,37969 & & & & & $93,96^{4}$ & 0,00788 & $20,46^{3}$ & 7 \\
\hline
\end{tabular}

em que: os números sobescritos se referem a nota alcançada pelo modelo nas respectivas estatísticas. $\mathrm{N}=$ somatório das notas.

Tabela 3. Notas obtidas pela classificação dos modelos de acordo com os resultados dos critérios estatísticos MDA, DPD e SQRR para cada classe de diâmetro.

Table 3. Notes obtained by classification of the models according to the results of the statistical criteria MDA, DPD and SQRR for each diameter class.

\begin{tabular}{|c|c|c|c|c|c|c|c|}
\hline & \multicolumn{5}{|c|}{ Classe de diâmetro } & & \\
\hline Modelo & 1 & 2 & 3 & 4 & 5 & $\mathrm{~N}$ & $\mathrm{R}$ \\
\hline 1 & 19 & 10 & 5 & 16 & 12 & 62 & 2 \\
\hline 2 & 9 & 13 & 17 & 19 & 22 & 80 & 8 \\
\hline 4 & 19 & 16 & 11 & 8 & 11 & 65 & 4 \\
\hline 7 & 22 & 18 & 15 & 6 & 4 & 65 & 4 \\
\hline 8 & 10 & 14 & 17 & 12 & 22 & 75 & 6 \\
\hline 12 & 4 & 11 & 11 & 21 & 14 & 61 & 1 \\
\hline 13 & 7 & 15 & 13 & 18 & 18 & 71 & 5 \\
\hline 15 & 19 & 18 & 25 & 12 & 5 & 79 & 7 \\
\hline 16 & 22 & 18 & 15 & 6 & 4 & 65 & 4 \\
\hline 18 & 13 & 3 & 14 & 18 & 16 & 64 & 3 \\
\hline
\end{tabular}

em que: $\mathrm{N}$ (nota) e R (rancking)= Soma do total das notas de cada classe diametrica e classificação do modelo, respectivamente, considerando as notas que cada modelo obteve nas diferentes classes. 
(A) - Modelo 1

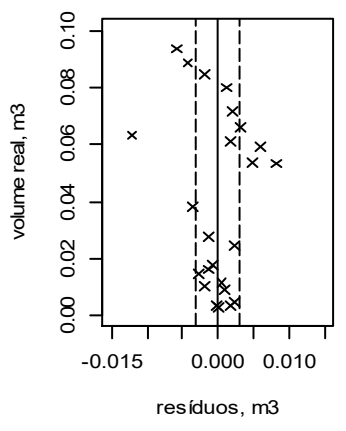

(D) - Modelo 12

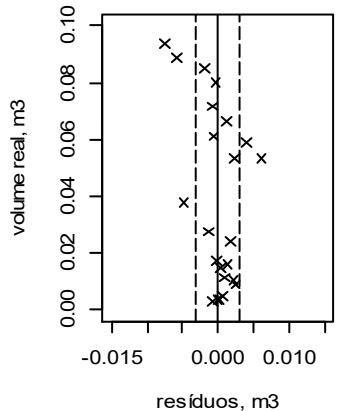

(B) - modelo 1

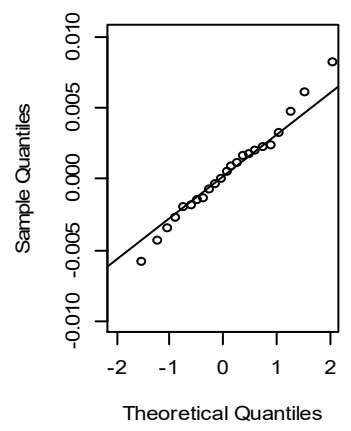

(E) - modelo 12

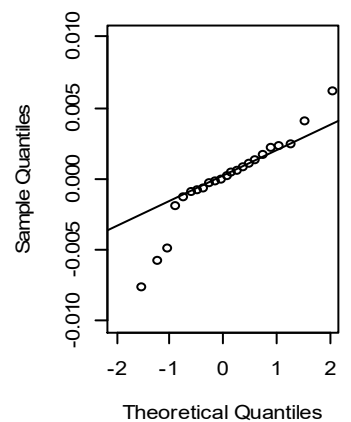

(C) - modelo 1

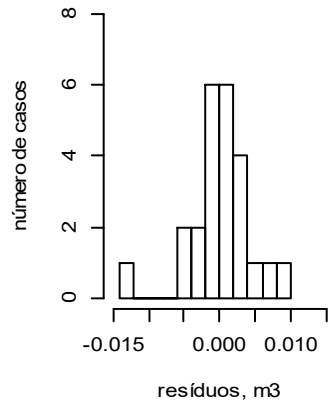

(F) - modelo 12

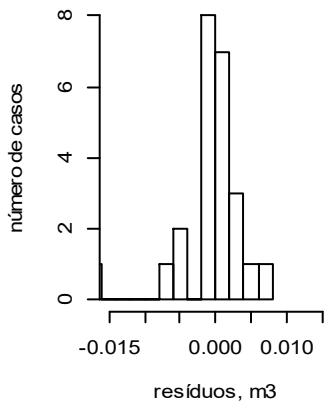

Figura 1. Distribuição de resíduos do volume com casca para os modelos 1 e $12\left(\mathrm{em} \mathrm{m}^{3}\right)$. Figure 1. Distribution of residues from husk volume for models 1 and $12\left(\text { in }^{3}\right)^{3}$.

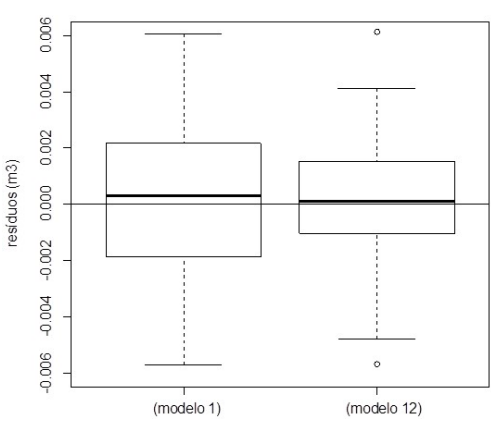

Figura 2. Valores residuais do volume com casca mínimo, primeiro quartil, mediana, segundo quartil e máximo obtidos para os modelos 1 e $12\left(\mathrm{em} \mathrm{m}^{3}\right)$.

Figure 2. Residual values of the volume with minimum bark, first quartile, median, according to quartile and maximum obtained for models 1 and 12 (in $\mathrm{m}^{3}$ ).

O emprego da variável binária Tx no modelo 12 (Leite et al., 1995), usando o volume com e sem casca na mesma base de dados, tendo $\mathrm{Tx}=0$ para volume com casca e $\mathrm{Tx}=1$ para volume sem casca, foi realizado a apartir do ajuste do modelo 12 na forma funcional: $\operatorname{Ln}(\mathrm{v})=\beta_{0}+\beta_{1} \operatorname{Ln}(\mathrm{d}+1)+$ $\beta_{2} \operatorname{Ln}(\mathrm{h})+\beta_{3} \mathrm{Tx}+\operatorname{Ln}(\varepsilon) \quad$ (27). Como resultado, foi gerada a seguinte equação volumétrica:

Volume com e sem casca (v)

$\hat{\mathrm{V}}=\mathrm{e}^{[1,286 \operatorname{Ln}(\mathrm{d}+1)+2,105 \operatorname{Ln}(\mathrm{h})-11,700-0,167(\mathrm{Tx})]} ; \quad \operatorname{comR}^{2} \mathrm{aj}=97,83 \%$ e syx $=12,03 \% \quad$ (Equação 3 )

$\mathrm{Na}$ Tabela 4 são apresentados os resultados obtidos pela aplicação das equações 1 e $3(\mathrm{Tx}=0)$ para obter o volume com casca e das equações 2 e $3(\mathrm{Tx}=1)$ para obter o volume sem casca. Pelas estatísticas MDA, SD e SQRR, nota-se que o uso da equação 3 resultou em primeiro lugar com 8 pontos.
Tabela 4. Resultados da classificação das equações 1, 2 e 3 na quantificação dos volumes com e sem casca.

Table 4. Results of the classification of equations 1,2 and 3 in the quantification of the volumes with and without shell.

\begin{tabular}{lccccc}
\hline Equação & $\mathrm{MDA}$ & $\mathrm{SD}$ & $\mathrm{SQRR}$ & $\mathrm{N}$ & $\mathrm{Nt}$ \\
\hline \multicolumn{7}{c}{ Volume com casca } \\
Equação 1 & $-0,00061^{2}$ & $0,00445^{2}$ & $0,373^{2}$ & 6 & $10^{2}$ \\
Equação 3; Tx=0 & $-0,00047^{1}$ & $0,00440^{1}$ & $0,367^{1}$ & 3 & $8^{1}$ \\
\hline \multicolumn{7}{c}{ Volume sem casca } \\
Equação 2 & $-0,00069^{1}$ & $0,00382^{1}$ & $0,822^{2}$ & 4 & \\
Equação 3; Tx=1 & $-0,00086^{2}$ & $0,00389^{2}$ & $0,408^{1}$ & 5 & \\
\hline
\end{tabular}

Para volume sem casca, ao comparar a equação 2 (Figuras 3A, 3B e 3C) com a equação 3 fazendo $\mathrm{Tx}=1$ (Figuras 3D, 3E e 3F), respectivamente, pode-se ver que a distribuição de resíduos foi similar. Quanto ao volume com casca, ao comparar a equação 1 (Figuras 1D, 1E e 1F) com a equação 3 fazendo $\mathrm{Tx}=0$ (Figuras $3 \mathrm{G}, 3 \mathrm{H}$ e $3 \mathrm{I}$ ), se observa que houve inexpressiva melhoria na distribuição dos resíduos para a equação 3 com $T x=0$, tendo $64 \%(16 / 25)$ de ocorrência destes em torno de $\pm 0,002 \mathrm{~m}^{3}$ (Figura 3I versus Figura $1 \mathrm{~F}$ ). Portanto, pode-se empregar a equação $3 \mathrm{em}$ árvores individuais do clone GG100 no sul do Tocantins.

\section{DISCUSSÃO}

Os resultados da Tabela 2 mostraram forte evidência sobre a importância da variável altura no ajuste de modelos volumétricos, incrementando expressiva melhoria com elevação do $\mathrm{R}^{2}$ aj para acima de 0,970 e syx reduzindo para menor que $12,5 \%$, se considerado o melhor modelo volumétrico regional (modelo 12).

Apesar do diâmetro ter uma expressiva importância na explicação da variável volume, certamente, a variável altura não deve ser negligênciada no uso de modelos volumétricos porque irá proporcionar um melhor grau de ajuste com o seu uso. Resultados similares a estes, também foram obtidos em 
outros trabalhos, como os conduzidos por: Leite; Andrade (2002), Rezende et al. (2006), Rolim et al. (2006), Machado et al. (2008), Pelissari et al. (2011), Miguel; Leal (2012).
Portanto, deve-se aceitar a hipótese $\mathrm{Ho}_{1}$, o que leva a preferência pelo ajuste de modelos volumétricos regionais para o clone GG100 no sul do Tocantins.
(A) - sem casca

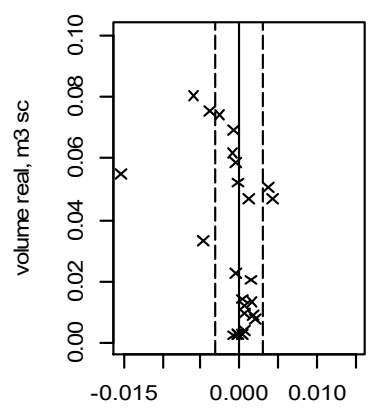

resíduos, $\mathrm{m} 3 \mathrm{sc}$

(D) $-T x=1$

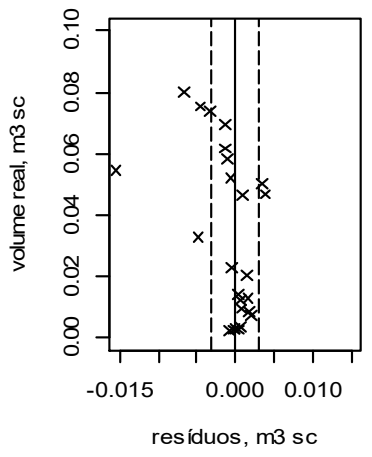

(G) $-T x=0$

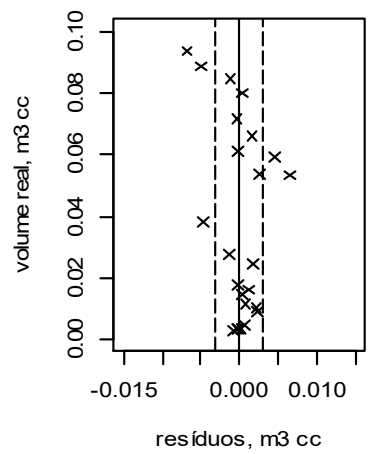

(B) - sem casca

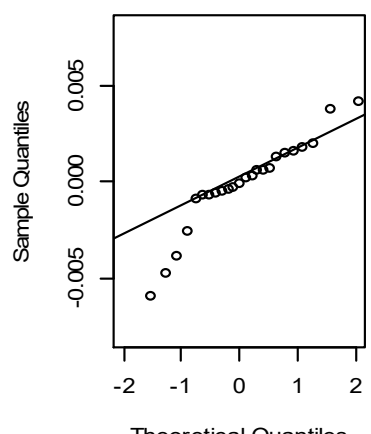

(E) $-T x=1$

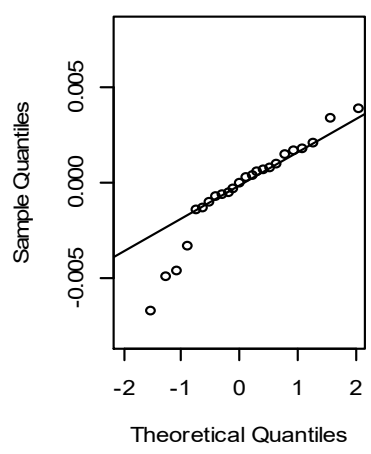

(H) $-T x=0$

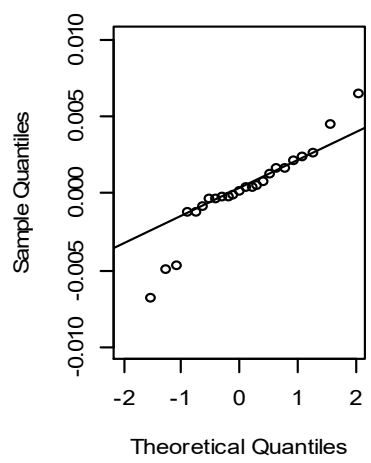

(C) - sem casca

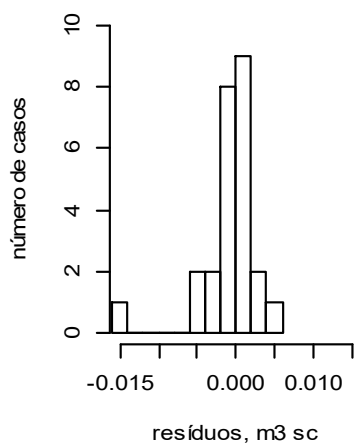

(F) $-T x=1$

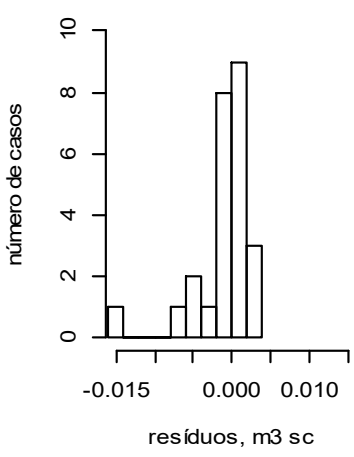

(I) $-T x=0$

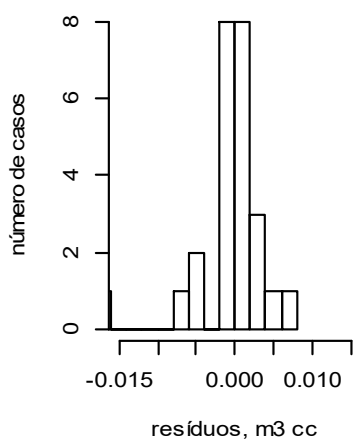

Figura 3. Distribuição de resíduos do volume sem casca, em $\mathrm{m}^{3}$ (A ao $\mathrm{C}$ - equação 2; D ao $\mathrm{F}$ - equação 3 com $\mathrm{Tx}=1$ ) e do volume com casca ( $\mathrm{G}$ ao I - equação $3 \mathrm{com} \mathrm{Tx}=0)$.

Figure 3. Distribution of residues from the shelled volume, in $\mathrm{m}^{3}$ (A to $\mathrm{C}$ - equation 2; $\mathrm{D}$ to $\mathrm{F}$ - equation 3 with $\mathrm{Tx}=1$ ) and the volume with bark ( $\mathrm{G}$ to $\mathrm{I}-$ equation 3 with $\mathrm{Tx}=0)$.

Ao se considerar os demais critérios de análises (Tabela 3, Figuras 1 e 2 e pressuposições da regressão), verificou-se que o modelo 12 se sobressaiu para uso no clone GG100. Neste caso, como na grande maioria dos inventários florestais, se quantificam o volume com e sem casca $\left(\mathrm{v}_{\mathrm{cc}}\right)$, então, se exige o ajuste de modelos volumétricos também para dados de volume sem casca $\left(\mathrm{v}_{\mathrm{sc}}\right)$. Neste caso, para cada situação inventariada, são geradas duas equações volumétricas em separado, sendo uma para volume com casca e outra para volume sem casca (Equações 1 e 2).

Ocorre que, dado à variação de diferentes situações de aplicabilidade das equações volumétricas (idade, espaçamento, local, solo, espécie, material genético), necessita-se o ajuste de uma grande quantidade das equações
1 e 2 para obter $\mathrm{v}_{c c}$ e $\mathrm{v}_{c c}$, respectivamente. Neste problema, o emprego da variável binária Tx no modelo 12 (Equação 3) demonstrou ser uma solução bastante viável (Tabela 4 e Figura 3 comparada a Figuras 1), pois, com um único ajuste, se obtem condições de quantificar tanto $\mathrm{v}_{\mathrm{cc}}$ como $\mathrm{v}_{\mathrm{cc}}$ com a mesma equação volumétrica. Isso permite uma redução em $50 \%$ do número de equações necessárias em um inventário florestal para obter $\mathrm{v}_{\mathrm{cc}} \mathrm{e} \mathrm{v}_{\mathrm{cc}}$. Portanto, com a aceitação da $\mathrm{H}_{02}$, se pode utilizar a variável binária Tx em mdelos volumétricos regionais ajustados para o clone GG100 no sul do Tocantins.

\section{CONCLUSÕES}

Diante dos resultados obtidos, pôde-se concluir pela aceitação da hipótese $\mathrm{Ho}_{1}$ levando a decisão em se preferir o 
ajuste somente de modelos volumétricos regionais para o clone GG100 no Tocantins.

Quanto a hipótese $\mathrm{Ho}_{2}$, pôde-se concluir pela sua aceitação. Isto levou a decisão pelo ajuste de uma única equação para quantificar, tanto o volume de madeira com casca como sem casca aplicando a variável binária Tx no modelo 12. Portanto, para quantificar tanto o volume com casca como o volume sem casca em árvores individuais do clone jovem GG100 no sul do Tocantins, concluiu-se pela seguinte volumétrica:

$$
\hat{\mathrm{V}}=\mathrm{e}^{[1,286 \operatorname{Ln}(\mathrm{d}+1)+2,105 \operatorname{Ln}(\mathrm{h})-11,700-0,167(\mathrm{Tx})]}
$$

com $\mathrm{Tx}=0$ para volume com casca e $\mathrm{Tx}=1$ para volume sem casca.

\section{REFERÊNCIAS}

ANDRADE, V. C. L. Modelos volumétricos de dupla entrada para aplicar em povoamentos florestais brasileiros. Floresta e Ambiente, Seropédica, v. 24, e00135415, 2017. DOI: http://dx.doi.org/10.1590/2179-8087.135415

ANDRADE, V. C. L.; SCHMITT, T. Modelos volumétricos de dupla entrada avaliados para Pinus caribaea var. hondurensis no município de Posse, Goiás. Revista Brasileira de Biometria, Lavras, v. 34, n. 4, p. 656-668, 2016.

ANDRADE, V. C. L.; CALEGARIO, N.; SCOLFORO, J. R. S. Análise de algumas alternativas para obter o coeficiente angular da reta no método da altura relativa. Ciência Florestal, Santa Maria, v. 16, n. 3, p. 303-317, 2006.

DOI: http://dx.doi.org/10.5902/198050981910

ANDRADE, V. C. L.; LEITE, H. G. Uso da geometria analítica para descrever o tapere quantificar o volume de árvores individuais. Revista Árvore, Viçosa, v. 25, n. 4, p. 481-486, 2001.

CAMPOS, J. C. C.; LEITE, H. G. Mensuração florestal: perguntas e respostas. 4. ed. Viçosa: Editora UFV, 2013. $605 \mathrm{p}$.

CAMPOS, J. C. C.; TREVIZOL JÚNIOR, T. L.; PAULA NETO, F. Ainda, sobre a seleção de equações de volume. Revista Árvore, Viçosa, v. 9, n. 2, p. 115-126, 1985.

COUTO, H. T. Z.; VETTORAZZO, S. C. Seleção de equações de volume e peso seco comercial para Pinus taeda. Cerne, Piracicaba, v. 5, n. 1, p. 69-80, 1999.

IBÁ_INDÚSTRIA BRASILEIRA DE ÁRVORES. Relatório 2017: ano base 2016. Brasília, DF, 2017. 80 p. Disponível em: $<$ http://iba.org/images/shared/Biblioteca/IBA_RelatorioA nual2017.pdf $>$. Acesso em: 03/09/2017.

IMAÑA-ENCINAS, J. et al. Equações de volume de madeira para o cerrado de Planaltina de Goiás. Floresta, Curitiba, PR, v. 39, p. 107-116, 2009.

LEITE, H. G.; ANDRADE, V. C. L. Um método para condução de inventários florestais sem o uso de equações volumétricas. Revista Árvore, Viçosa, v. 26, n. 3, p. 321 328, 2002. DOI: http://dx.doi.org/10.1590/S010067622002000300007

LEITE, H. G.; GUIMARÃES, D. P.; CAMPOS, J. C. C. Descrição e emprego de um modelo para estimar múltiplos volumes de árvores. Revista Árvore, Viçosa, v. 19, n. 1, p. 1-21, 1995
MACHADO, S. A; FIGUEIREDO-FILHO, A. Dendrometria. 2 ed. Guarapuava: Unicentro, 2006. $316 \mathrm{p}$.

MACHADO, S. A.; CONCEIÇÃO, M. B.; FIGUEIREDO, D. J. Modelagem do volume individual para diferentes idades e regimes de desbaste em plantações de Pinus oocarpa. Revista Ciências Exatas e Naturais, Curitiba, v. 4, p. 185-197, 2002.

MACHADO, S. A.; FIGURA, M. A.; SILVA, F. C. R. da; TÉO, S. J.; STOLLE, L.; URBANO, E. Modelagem volumétrica para bracatinga (Mimosa scabrella) em povoamentos da região metropolitana de Curitiba. Pesquisa Florestal Brasileira, Colombo, n. 56, p. 17-29, 2008.

MIGUEL, E. P.; LEAL, F. A. Seleção de equações volumétricas para a predição do volume total de Eucalyptus urophylla s. t. blake na região norte do Estado de Goiás. Enciclopédia Biosfera, Goiânia, v. 8, p. 13721386, 2012.

PELISSARI, A. L.; LANSSANOVA, L. R.; DRESCHER, R. Modelos volumétricos para Pinus tropicais, em povoamento homogêneo, no Estado de Rondônia. Pesquisa Florestal Brasileira, Colombo, v. 31, p. 173181,2011

REGAZZI, A. J.; SILVA, C. H. O. Teste para verificar a igualdade de parâmetros e a identidade de modelos de regressão não-linear. I. Dados no delineamento inteiramente casualizado. Revista de Matemática e Estatística, São Paulo, v. 22, n. 3, p. 33-45, 2004.

R CORE TEAM. R: a language and environment for statistical computing. Disponível em: <http://www.rproject.org $>$. Acesso em: $10 \mathrm{dez} 2015$.

REZENDE, A. V.; VALE, A. T. do; SANQUETTA, C. R.; FIGUEIREDO FILHO, A.; FELFILI, J. M. Comparação de modelos matemáticos para estimativa do volume, biomassa e estoque de carbono da vegetação lenhosa de um cerrado sensu stricto em Brasília, DF. Scientia Forestalis, Brasília, n. 71, p. 65-76, 2006.

ROCHA, T. B.; CABACINHA, C. D.; ALMEIDA, R. C. de; PAULA, A. de; SANTOS, R. C. Avaliação de métodos de estimativa de volume para um povoamento de Eucalyptus urophylla s. T. Blake no Planalto da Conquista- BA. Enciclopédia Biosfera, Goiânia, v. 6, p. $1-13,2010$

ROLIM, S. G; COUTO, H. T. Z. do; JESUS, R. M. de; FRANÇA, J. D. Modelos volumétricos para a Floresta Nacional do Tapirapé-Aquirí, Serra dos Carajás (PA). Acta Amazonica, Manaus, v. 36, p. 107-114, 2006. DOI: http://dx.doi.org/10.1590/S0044-59672006000100013

SCHNEIDER, P. R.; SCHNEIDER, P. S. P.; SOUZA, C. A. $M$. Análise de regressão aplicada à engenharia florestal. Santa Maria: FACOS-UFSM, 2009. 294 p.

SEPLAN_Secretaria do Planejamento e da Modernização da Gestão Pública. Atlas do Tocantins: subsídios ao planejamento da gestão territorial. 6. ed. Palmas: Seplan, 2012. $80 \mathrm{p}$.

VEIGA, R. A. A.; CARVALHO, C. M.; BRASIL, M. A. M. Determinação de equações de volume para árvores de Acacia mangium Willd. Cerne, Lavras, v. 6, n. 1, p. 103$107,2000$. 